\title{
Interchain Electronic Excitations in Poly(phenylenevinylene) (PPV) Aggregates
}

\author{
S. Tretiak,* A. Saxena, R. L. Martin, and A. R. Bishop \\ Theoretical Division and Center for Nonlinear Studies, Los Alamos National Laboratory, \\ Los Alamos, New Mexico 87545 \\ Received: February 1, 2000; In Final Form: May 8, 2000
}

\begin{abstract}
The semiempirical collective electronic oscillators (CEO) approach based on a time-dependent HartreeFock approximation is applied to analyze formation of interchain electronic excitations in PPV aggregates composed of two weakly coupled chain segments. Real-space analysis of transition density matrices identifies the origins of electron-hole pairs created upon optical excitation. The singlet intrachain excitons are delocalized along the whole oligomer chain and manifest themselves as strong band edge transitions in the linear spectra. New low-frequency interchain electronic excitations appear upon bringing oligomer chains into close proximity (3-4 $\AA$ ). These new electronic states localized at the near-contact have no signatures in linear absorption. We argue that they may significantly decrease luminescence quantum efficiency of polymer films.
\end{abstract}

\section{Introduction}

Emissive conjugated polymers based on the poly(phenylenevinylene) (PPV)-like structures have attracted significant attention due to their electronic and optical properties which make them suitable materials for device applications. Potential technological applications include electroluminescent, ${ }^{1-3}$ photovoltaic, ${ }^{4}$ and optoelectronic ${ }^{5-7}$ devices, photodetectors, ${ }^{8,9}$ transistors, ${ }^{10,11}$ and solid-state lasers. ${ }^{12-14}$ Luminescence quantum efficiency is a crucial issue for the successful design of such systems. The major problem in achieving high luminescence quantum yields is believed to be aggregation quenching of the emissive state caused by interchain interactions. ${ }^{15-20}$ Use of block copolymers, ${ }^{21}$ polymer blends, ${ }^{19}$ and polymers with attached bulky side groups $s^{22,23}$ have been suggested to reduce the aggregation.

A fundamental understanding of the photophysics in a unified framework is needed for successful development of the above technology. Extensive experimental studies have addressed the effects of aggregation on the photophysical properties of polymer materials. Transient absorption spectroscopy experiments have assigned various spectroscopic features to spatially indirect excitons or bound polaron pairs, ${ }^{18,19,24,25}$ excimers and exciplexes, ${ }^{17,26}$ biexcitons, ${ }^{27,28}$ and hole polarons, ${ }^{29}$ etc. Siteselective fluorescence experiments ${ }^{30}$ and photoluminescence quantum efficiency measurements ${ }^{31}$ suggest that over $90 \%$ of the initial photoexcitations in solid-state PPV samples are singlet intrachain excitons, ${ }^{30,31}$ whereas other authors have assigned the majority of the initial photoexcitations to interchain species. ${ }^{17,24}$

Although one-dimensional semiconductor band models ${ }^{32-34}$ and quantum-chemical computations ${ }^{35-39}$ have both proven to be useful tools for interpretation of the photophysical properties of conjugated polymers, the impact of interchain interaction has not yet been extensively studied theoretically. In view of the incomplete knowledge of the chain packing, the theoretical challenge in evaluating these interactions, and the computational difficulties, only a few investigations have been devoted to the interchain effects on the polymer electronic structure. The interchain exciton states have been studied in refs 40 and 41 within a simple tight-binding model. The excimer formation

* Corresponding author. E-mail: serg@cnls.lanl.gov. and its effect on the polymer photoluminescence has been modeled using a simplified microscopic tight-binding Hamiltonian. ${ }^{42,43}$ The exciton splitting of two stilbene molecules has been addressed in ref 44 using an AM1 semiempirical treatment. The effects of relative molecular orientations on the energies and oscillator strengths of the lowest excited states in conjugated polymer dimers have been investigated in ref 45 . However, the last study was only conducted for large separations ( $4 \AA$ ) where intermolecular charge transfer is small. Monte Carlo simulations of the chain packing in the different PPV derivatives resulted in 3.3-4.2 $\AA$ interchain separations. ${ }^{46}$ An experimental/theoretical study of the family of stilbenoid dimers bound to a paracyclophane core showed that new interchromophore states with strong electron exchange appear at smaller distances. ${ }^{47}$ These states significantly affect absorption/emission properties of molecules. ${ }^{47}$ These results ${ }^{46,47}$ clearly indicate the necessity of further investigation of the dependence on the distance between chromophores, and how this affects the electronic structure of the aggregate.

In the current paper we conduct a systematic theoretical investigation of these interaction effects on the low-frequency electronic structure of a PPV dimer using the collective electronic oscillator (CEO) approach, ${ }^{37,47-49}$ which allows us to interpret the electronic excitations in terms of underlying electron-hole motion. The dimer consists of two identical cofacial large oligomers, which closely mimic the long-chain limit. We explore different intermolecular orientations and compare the principal physical phenomena. The study is focused on the transition from long-range dipole-dipole electrostatic coupling to the strong electron exchange interaction which dominates in aggregates with close contact. We show that any contact closer than $\sim 4 \AA$ leads to the formation of low-lying intermolecular states which may account for reduced photoluminescence in these materials.

Section II A briefly describes the computational method. In section II B we analyze the electronic structure of the isolated monomer. In sections II C-II E we investigate the effect of the dimer interactions on linear absorption and the relevant electronic modes of the dimers, and link their properties to the corresponding modes of the monomers. Finally we discuss the trends that emerge and summarize our results in section III. 


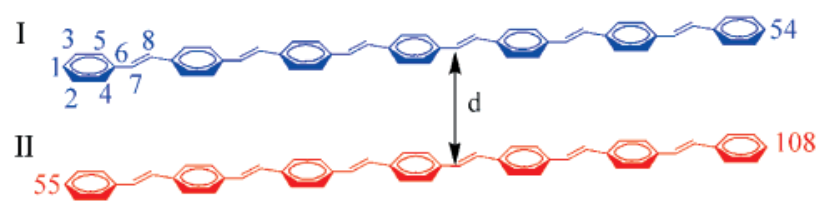

Case 1

a)

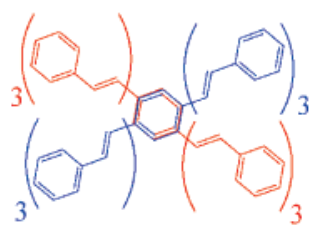

b)

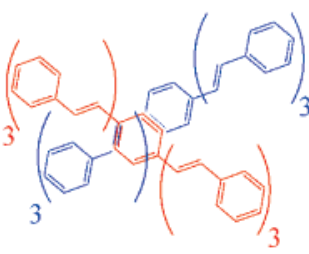

Case 2

a)

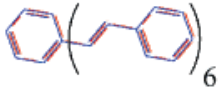

b)

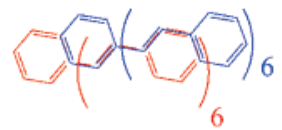

a)

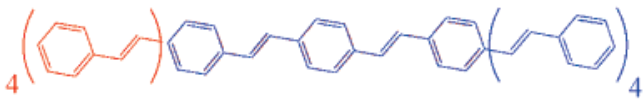

Case 3

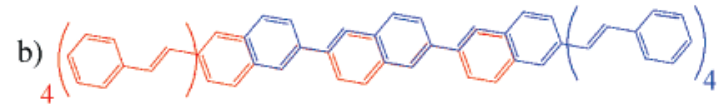

Figure 1. Top panel: Structure and atom labeling of oligo-PPV dimer (side view). The interchain distance $d$ has been varied (3-10 $\AA$ ) in CEO calculations. Different intermolecular orientations are shown in case 1 through case 3 molecular templates for phenyl-phenyl (a) and phenyl-vinyl interaction (b) (top views). Case 1 displays single unit (minimal) contact. Cases 2 and 3 represent the whole molecule (maximum) and three-unit (intermediate) contacts, respectively.

\section{Real-Space Analysis of Electronic Excitations}

A. Computational Method. To create various intermolecular orientations we first build PPV oligomers of varying length (e.g., molecule I in Figure 1 represents a PPV chain with seven repeat units). Each structure was then optimized at the Austin Model 1 (AM1) semiempirical level ${ }^{50}$ which provides a reasonable ground-state geometry of the polymer chain. In the course of optimization the molecular geometries were restricted to be planar. For reference, we also optimized these oligomers without constraining their geometries. (The optical properties of both planar and nonplanar oligomers are compared in section II B.) We then use the seven repeat unit (PPV-7) oligomer planar structure to create dimers consisting of two identical molecules at varying interchain separation $d=3-10 \AA$ with $0.1 \AA$ step (top structure in Figure 1). These were then used for consequent excited state electronic structure calculations without any further geometry optimization. The spectroscopic observables of PPV-7 are essentially saturated and well mimic the long-chain limit. ${ }^{37}$ The CEO electronic structure computations of PPV-7 dimer is not very expensive $\mathrm{e}^{4,51,52}$ (it takes $\sim 5 \mathrm{~h}$ to compute the 30 lowest excited states of PPV-7 dimer on the single CPU of Intel Pentium III $450 \mathrm{MHz}$ workstation), and larger chains are readily accessible. We consider three different intermolecular orientations shown in Figure 1. Case 1 corresponds to minimal interaction $\left(60^{\circ}\right.$ angle between polymer chains) when only single phenyl-phenyl (a) or phenyl-vinyl (b) groups of monomers are coupled. Case 2 describes the possible maximum interaction: Both chains are parallel and the whole molecule is coupled. Finally, case 3 shows an intermediate interaction situation, where only three repeat units of each monomer are involved in the contact.

We next applied the numerical CEO approach for calculating electronic structure. This method has been described in detail elsewhere. ${ }^{48,51}$ The ZINDO code was first applied to generate the intermediate neglect of differential overlap/spectroscopy (INDO/S) Hamiltonian ${ }^{53-56}$ using the geometries of our molecules and aggregates. We next calculated the Hartree-Fock ground-state density matrices ${ }^{57,58}$ which are the input to the subsequent CEO calculation. The CEO procedure ${ }^{48,51}$ was applied to compute 30 lowest excited-state frequencies and the relevant transition density matrices (denoted the electronic normal modes, $\xi_{v}$ ), which connect molecular optical response with the underlying electronic motions. Each mode is a matrix representing the electronic transition between the ground state $|g\rangle$ and an electronically excited state $|v\rangle$. Its matrix elements are given by

$$
\left(\xi_{v}\right)_{m n}=\left(v\left|c_{m}^{+} c_{n}\right| g\right\rangle
$$

where $c_{m}^{+}\left(c_{m}\right)$ are creation (annihilation) operators of an electron at the $m$ th atomic orbital, and $|g\rangle(|v\rangle)$ is the ground (excited) state many-electron wave function. The modes can be computed as eigenmodes of the linearized time-dependent Hartree-Fock (TDHF) equations of motion for the density matrix driven by the external field, totally avoiding the explicit calculation of many-electron excited-state wave functions. The eigenfrequencies $\Omega_{v}$ of these equations provide the optical transition energies. ${ }^{48,51}$ The numerical effort involved in computing these eigenvalues and eigenvectors is greatly reduced by using the oblique Lanczos algorithm. ${ }^{52}$ Transition dipole moments $\mu_{v}=\operatorname{Tr}\left(\mu \xi_{v}\right)$ were then calculated using the dipole moment operator $\mu=\sum_{n m} \mu_{n m} c_{m}^{+} c_{n}$. Then $f_{v}=2 \Omega_{v} \mu_{v}^{2}$ is the oscillator strength of the $g$ to $v$ transition.

The electronic modes represent collective motions of electrons and holes and carry substantially less information than the complete many-electron eigenstates but more than required for calculating molecular polarizabilities and spectroscopic observables. The diagonal elements $\left(\xi_{v}\right)_{n n}$ represent the net charge induced on the $n$th atomic orbital by an external field with frequency $\Omega_{v}$, whereas $\left(\xi_{v}\right)_{m n} n \neq m$ is the dynamical bondorder (coherence) representing the joint amplitude of finding an electron on orbital $m$ and a hole on orbital $n$. The INDO/S Hamiltonian assigns a single $s$-type basis function to hydrogen atoms and four basis functions $\left(s, p_{x}, p_{y}, p_{z}\right)$ to the carbon atoms of a PPV oligomer. The orbitals $s, p_{y}$, and $p_{z}$ on the carbons in the chain are $s p^{2}$ hybridized and form the molecular $\sigma$-bonding skeleton. Qualitatively, only the $p_{x}$ orbitals perpendicular to the molecular plane participate in the $\pi$-bonding network and are responsible for the lowest optical excitations.

To represent the contribution of all atomic orbitals on each carbon to the transition density we have used the following contraction. The total induced charge on each atom $\mathrm{A}$ is given by the diagonal elements as

$$
\left(\xi_{v}\right)_{A}=\sum_{n_{A}}\left(\xi_{v}\right)_{n_{A} n_{A}}
$$

whereas an average over the off-diagonal elements represents the effective coherence between atoms A and B.

$$
\left(\xi_{v}\right)_{\mathrm{AB}}=\sqrt{\sum_{n_{\mathrm{A}} m_{\mathrm{B}}}\left[\left(\xi_{v}\right)_{n_{\mathrm{A}} m_{\mathrm{B}}}\right]^{2}}
$$

Here the indices $n_{\mathrm{A}}$ and $m_{\mathrm{B}}$ run over atomic orbitals localized 


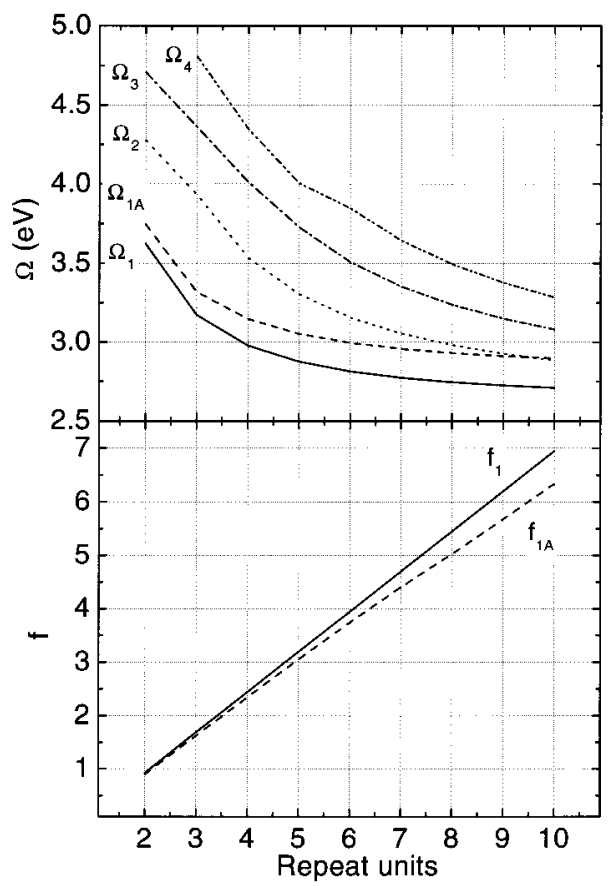

Figure 2. Variation of the four lowest excited-state energies (top panel) and lowest frequency (band-gap) transition oscillator strengths (bottom panel) with the number of repeat units of the PPV chain. AM1 optimized planar structures have been used for CEO input, except $\Omega_{1 \mathrm{~A}}$ and $f_{1 \mathrm{~A}}$ which correspond to a nonplanar optimized geometry (see text).

on atoms $\mathrm{A}$ and $\mathrm{B}$, respectively. The size of the matrix $\left(\xi_{v}\right)_{\mathrm{AB}}$ is now equal to the number of carbons in the molecular system. A two-dimensional representation of the electronic modes $\left(\xi_{v}\right)_{\mathrm{AB}}$ allows one to interpret and visualize these collective electronic motions in terms of the electronic density matrix in real space. ${ }^{37,47,49,59}$ The coordinate axes label atoms as given, for example, in the top panel of Figure 1, and hole and electron dynamics is shown along the $x$ and $y$ axes, respectively.

A detailed real-space analysis of electronic excitations in single-chain PPV oligomers was conducted in ref 37 using a Pariser-Parr-Pople (PPP) Hamiltonian ${ }^{60}$ which considers only one basis function per carbon atom (the $\pi\left(p_{x}\right)$ orbital). In the present article we use a more robust and accurate INDO/S Hamiltonian ${ }^{53-56}$ which also includes the orbitals composing the $\sigma$-framework. This necessitates a basis set which is $\sim 5$ times larger than PPP's basis set and significantly increases the computational memory and time requirements. For diagonalization of Liouville operator, the density-spectral-moment algorithm (DSMA) ${ }^{51}$ used in ref 37 focuses solely on the dominant peaks of the absorption spectrum. Here, we use the oblique Lanczos algorithm ${ }^{52}$ which computes accurately both active and forbidden transition energies and is most suited for our purpose. However, since the nature of the $\pi$-electronic excitations is qualitatively independent of the choice of the Hamiltonian, we further refer the reader to ref 37 for a more detailed electronic spectrum analysis of the single PPV oligomer. The main features of the spectrum are briefly outlined in the next section.

B. Single PPV chain (monomer). To establish the background for exploring interchain interactions, we first consider the electronic structure of a single planar PPV-7 oligomer, which is the building block of all our dimers studied. The top panel of Figure 2 shows the variation with oligomer size of energies corresponding to the four lowest electronic transitions. The energy of the lowest band-gap transition 1 (solid line) is responsible for a very strong absorption peak and saturates to
$2.7 \mathrm{eV}$ for long chains, which compares well with the experimental value $\sim 2.5 \mathrm{eV}$. Its oscillator strength, shown in the lower panel by a solid line, grows nearly linear with the system size. The higher frequency transitions saturate at a slower rate going toward the lowest excitation saturated limit. All four excited states have $\mathrm{B}_{\mathrm{u}}$ symmetry. For comparison we have also plotted the variation of the band-gap energy $\left(\Omega_{1 \mathrm{~A}}\right)$ and oscillator strength $\left(f_{1 \mathrm{~A}}\right)$ for the nonplanar oligomers. Interaction of the $\sigma$-bonds connecting neighboring phenyl-vinyl groups results in the structure where the neighboring phenyls are twisted by $\sim 17^{\circ}$. This deviation from the planar geometry reduces $\pi$-electron delocalization. Therefore, the band-gap energy saturates to a higher $2.9 \mathrm{eV}$ limit, and the oscillator strength is smaller as compared to the planar structures (dashed lines in Figure 2). However, these changes are small and there is no qualitative impact on the nature of the optical excitations.

To trace the origin of the various peaks we have examined the corresponding collective electronic modes of a planar PPV-7 oligomer. Two-dimensional plots of the matrices $\xi_{v} 37,47-49,59$ establish a direct link between the optical response and the underlying photoinduced real-space dynamics of charges. The matrix size is equal to the number of carbon atoms, labeled according to the top panel in Figure 1.

To establish a reference point, panel $\rho$ in Figure 3 shows the ground state density matrix of PPV-7. This matrix is diagonally localized, reflecting the nearest-neighbor chemical bonding in the ground state. Seven phenyl rings are clearly distinguishable. Panel $\Omega_{1}$ shows the band-edge transition 1 and is very similar to that calculated with the PPP Hamiltonian. ${ }^{37}$ This picture shows that the electron-hole created upon optical excitation is delocalized over the whole chain (diagonal in the plot) and tends to be in the middle of the molecule. The exciton size (maximal distance between electron and hole) is about $4-5$ repeat units (largest off-diagonal extent of the nonzero matrix area). Panel $\Omega_{2}$ displays the next transition 2 . This mode is forbidden in linear absorption and does not show up in the DSMA/PPP calculations. ${ }^{37}$ It has the same off-diagonal coherence size as mode 1 , but a nonuniform diagonal space distribution. The molecule is effectively broken into two parts with sizes of 3 repeat units and a very small electronic coherence between them. The electron-hole pair is located either in the first or in the second half of the chain, but not in the center. Two contributions to the transition dipole cancel each other, resulting in a vanishing oscillator strength. The next transition 3 shown in panel $\Omega_{3}$ is broken into the three parts. As shown in ref 48, the total contribution from the ends is approximately zero, and only the middle region contributes to the oscillator strength of this mode. This transition therefore makes only a weak contribution to the linear absorption. The molecule is effectively a trimer with weak electronic coherence among its parts. The off-diagonal coherence size is about 3 repeat units and the diagonal sizes are 2, 3, and 2 repeat units. The next mode (panel $\Omega_{4}$ ) is broken into four parts and has a vanishing transition dipole since the contributions from the sub-quarters cancel each other. Finally the transitions in the higher frequency spectrum $(3.5-7 \mathrm{eV})$ become localized to a single repeat unit and further to a single phenyl and a single vinyl group. ${ }^{37}$ For example, the panel $\Omega_{\mathrm{L}}$ electronic mode (forbidden in linear absorption) corresponds to the exciton completely localized on the central phenyl of the chain.

Thus the general trend of the electronic modes with increasing frequency is an effective aggregation of the molecule to small segments with weak electronic coherence among them. This is universal for larger polymers. The higher frequency modes tend to have more diagonal nodes. ${ }^{37,48,51}$ The number of nodes is 

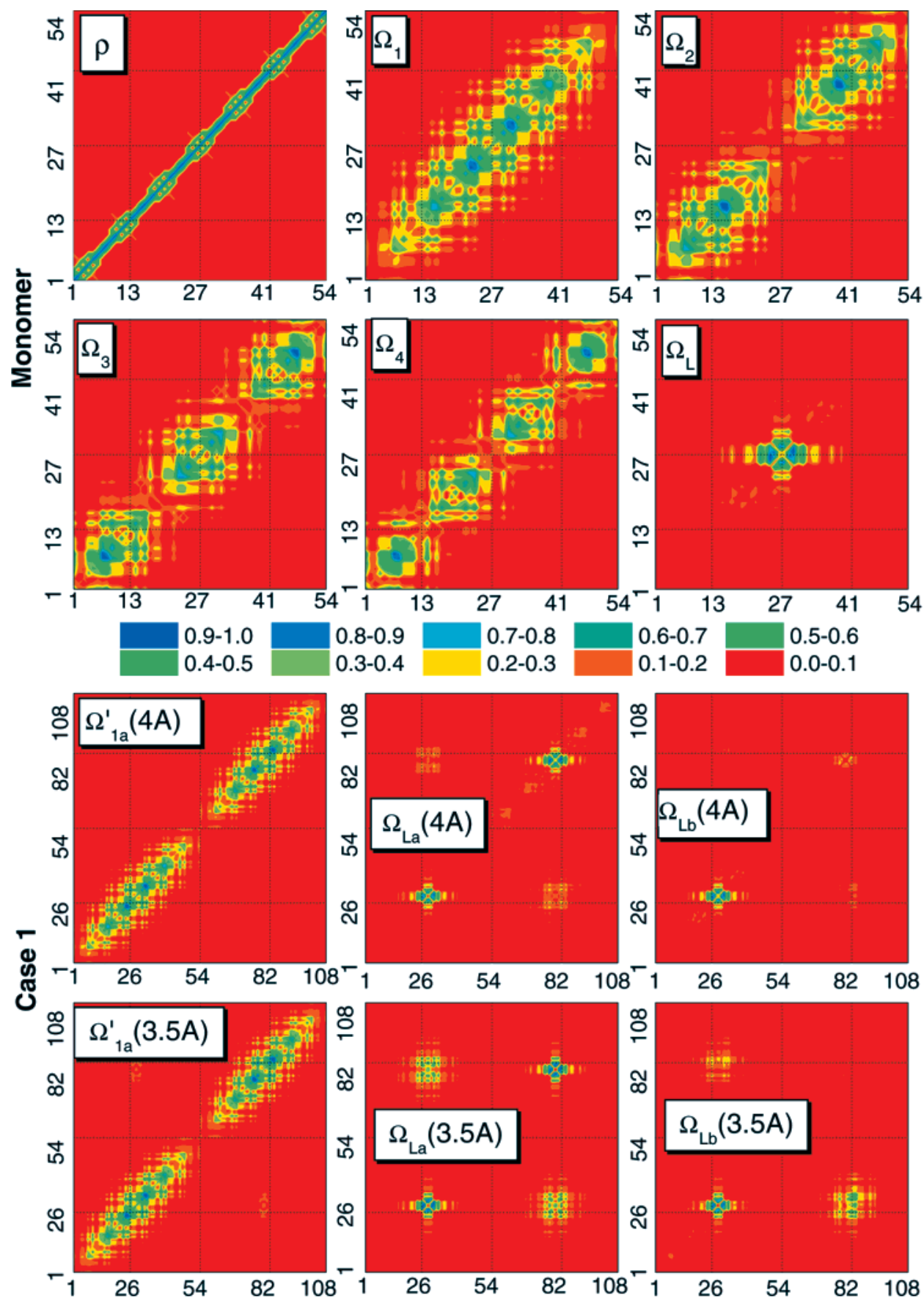

Figure 3. Contour plots of the electronic modes of a PPV-7 monomer (top two rows) and PPV dimer for case 1 (bottom two rows). The axis labels represent the individual atoms, as shown in Figure 1. The color map is given in the middle row. The monomer mode frequencies $\Omega_{1}-\Omega_{4}$ are 2.77 , 3.05, 3.35, $3.64 \mathrm{eV}$ (also shown in Figure 2), and $\Omega_{\mathrm{L}}=3.99 \mathrm{eV}$. Panels $\rho$ and $\Omega_{\mathrm{L}}$ display the ground-state density matrix and mode of localized transition, respectively. The dimer modes are labeled according to Figures 1 and 4 . Their frequencies are $\Omega_{1 a}^{\prime}=2.76 \mathrm{eV}, \Omega_{\mathrm{L} a}=3.55 \mathrm{eV}, \Omega_{\mathrm{L} b}=$ $3.96 \mathrm{eV}$ (4 A separation) and 2.74, 2.72, $3.61 \mathrm{eV}(3.5 \AA$ A separation), respectively.

$n-1, n$ being the mode number in the energy hierarchy (transition $n$ in our notations). The mutual cancellation of the transition dipoles leads to vanishing oscillator strength of electronic modes with odd number of nodes, whereas the oscillator strength of electronic modes with even number of nodes scales as $\sim 1 / n^{2}$. For example, the intensity of third transition with two nodes is about nine times weaker than that of the band gap $1 \mathrm{~B}_{\mathrm{u}}$ state. All electronic modes are almost symmetric with respect to the diagonal $\left(\xi_{m n} \approx \xi_{n m}\right)$. This means that there is no preferred direction of motion for electrons (or holes). The electron-hole separation does not exceed 5 repeat units for all transitions, and the PPV-7 oligomer, therefore, well reflects the long chain limit.
C. PPV Dimer: case 1 (minimal contact). It is well understood that when the chromophores are well separated in space, their interaction is electrostatic (i.e., electron exchange or hopping is negligible). Each chromophore then retains its own electrons and the system may be described by a simple Frenkel exciton model common in molecular crystals and aggregated systems. ${ }^{61-64}$ Each monomer peak splits into two transitions in the dimer spectra and their wave functions are symmetric and antisymmetric combinations of the monomeric excited states wave functions. This Davydov splitting ${ }^{65,66}$ reflects interaction between chromophores and may be used to build an effective Hamiltonian of the system. ${ }^{67}$ Throughout this paper we will use the notation $\Omega^{\prime}$ and $\Omega^{\prime \prime}$ for this pair of states, where 


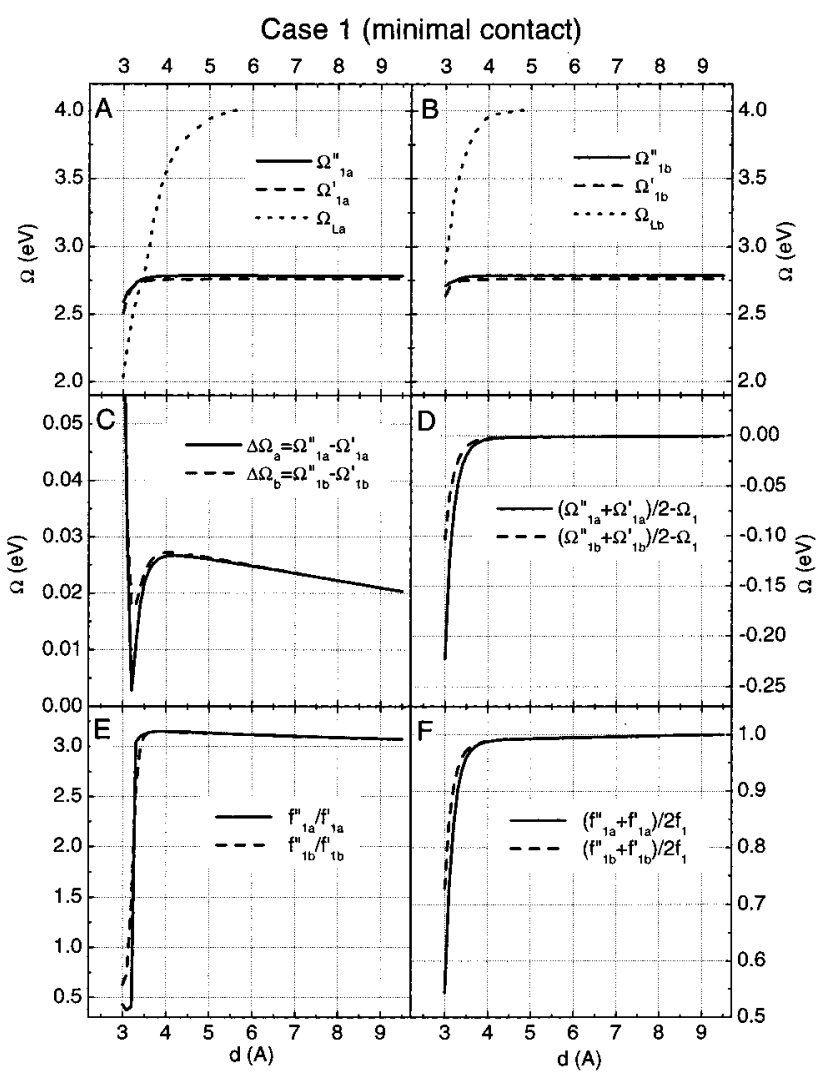

Figure 4. Variation of excited-state energies of delocalized $\left(\Omega_{1}^{\prime}, \Omega_{1}^{\prime}\right)$ and localized $\left(\Omega_{L}\right)$ transitions (panels A and B, respectively); splitting and position of center-of-mass for band-gap pair of dimer excited states (panels C and D); relative distribution and total oscillator strengths for band-gap pair of dimer excited states (panels $\mathrm{E}$ and $\mathrm{F}$ ) with separation of PPV chains for (a) and (b) structures of case 1 (Figure 1).

$\Omega^{\prime \prime}$ corresponds to the state with stronger oscillator strength.

However, the short-range electron exchange interaction becomes dominant when the distance between the chromophores is small. This strong interaction leads to charge transfer (CT) between molecules and formation of new delocalized electronic excitations. This general behavior has been understood for some time. In what follows, we will see the transition from Frenkel exciton to CT character occurs at an interoligomer distance of $\sim 4 \AA$.

We first analyze large chromophore separations $d \gtrsim 4 \AA$. Figure 4A shows the variation of frequencies in case 1(a) dimer pair, corresponding to the band gap transition $\left(1 \rightarrow 1 a^{\prime}, 1 a^{\prime \prime}\right)$. Here, energies of $\Omega_{1 a}^{\prime}$ and $\Omega_{1 a}^{\prime \prime}$ are very close to the corresponding monomer energy $\Omega_{1 a}$. Their splitting $\Delta \Omega_{1 a}$ shown in Figure $4 \mathrm{C}$, as expected, increases with decreasing separation between chains, indicating a stronger interaction between monomer transition dipoles. Figure 4D shows that the center of mass of $\Omega_{1 a}^{\prime}$ and $\Omega_{1 a}^{\prime \prime}$ is equal to the monomer frequency $\Omega_{1}$. The oscillator strength ratio $f_{1 a}^{\prime \prime} / f_{1 a}^{\prime}=3=\tan ^{2} 60^{\circ}$ (Figure 4E) reflects the intermolecular orientation and the total oscillator strength is conserved (Figure 4F). The variation of the frequencies and oscillator strengths for case 1(b) dimer shown in Figures $4 \mathrm{~B}-\mathrm{F}$ is very similar to that of case 1(a). The properties described above follow from the Frenkel exciton model for this simple aggregate.

To follow the evolution of the excited states at close intermolecular distances $d<4 \AA$, we need to analyze the electronic modes in real-space which provides a convenient method for identifying the electronic transitions. Atom labeling runs over monomer I first then over monomer II, following the top panel of Figure 1. At $d=4 \AA 1 a^{\prime}$ (panel $\Omega_{1 a}^{\prime}$ (4A) of Figure $3)$ is simply the superposition of the monomeric 1 states (compare to $\Omega_{1}$ ). The corners of the plot represent the monomers and there is no off-diagonal coherences between them. A new, much stronger coherent interaction between chromophores appears at closer separations when intermolecular electron exchange becomes important. At $d=3.5 \AA$ the $1 a^{\prime}$ electronic mode (panel $\Omega_{1 a}^{\prime}(3.5 \mathrm{~A})$ of Figure 3 ) shows weak off-diagonal coherences between molecules. The behavior of $1 a^{\prime \prime}$ electronic mode (not shown) is very similar to $1 a^{\prime}$. This coherent interaction leads to crucial changes in the spectrum and completely invalidates the simple exciton model. The frequency becomes significantly red-shifted (Figure 4A and D) since the exciton is delocalized over the whole system, i.e., processes with electron and hole on different molecules are allowed. $\Delta \Omega_{1 a}$ decreases first ( $\Omega_{1 a}^{\prime}$ and $\Omega_{1 a}^{\prime \prime}$ trade places), then rapidly increases at smaller $d$. The ratio $f_{1 a}^{\prime \prime} / f_{1 a}^{\prime}=3$ is no longer valid and the total oscillator strength is not conserved (Figure $4 \mathrm{E}-\mathrm{F}$ ). For case $1(\mathrm{~b})$ dimer (Figure $4 \mathrm{~B}-\mathrm{F}), 1 b^{\prime}$ and $1 b^{\prime \prime}$ show similar behavior.

A prominent feature of the dimer spectrum is evolution of the $\mathrm{L}$ mode (panel $\Omega_{\mathrm{L}}$ of Figure 3 ) localized on the central phenyl. It is clear that L located at the contact should have the largest coherent interaction with its counterpart on the next molecule. Indeed, at $d=4 \AA 1 \mathrm{~L} a$ (panel $\Omega_{\mathrm{L} a}(4 \mathrm{~A})$ of Figure 3 ) already has noticeable coherence and its frequency is therefore red-shifted by $0.5 \mathrm{eV}$ compared to $\Omega_{\mathrm{L}}$. Here, $\Omega_{\mathrm{L} a}$ rapidly falls with decreasing intermolecular separation and it becomes the lowest mode in the electronic spectrum for $d \leq 3.5 \AA$ (Figure 4A). This red-shift is attributed to a significant intermolecular electron exchange and associated coherence (panel $\Omega_{\mathrm{L} a}(3.5 \mathrm{~A})$ of Figure 3). Similar transitions localized on the two phenyls were computed with the CEO approach and observed experimentally in the spectra of the family of paracyclophane dimers. ${ }^{47}$ Formation of such low-energy states in locations where PPVchain have close contacts (e.g., films), leads to effective fluorescence quenching. ${ }^{17-19}$ Instead of emitting, excitons migrate to these low-energy traps which are nonemissive since L $a$ has a vanishing oscillator strength. It is interesting to notice that $\mathrm{L} a$ does not mix with the $1 a$ states, even at $3.5 \AA$ when $\mathrm{L} a$ and $1 a^{\prime \prime}$ states are near degenerate. This may be attributed to the nearly orthogonal nature of these states.

Even though $\mathrm{L}_{b}$ in case 1 (b) shows behavior analogous to $\mathrm{L}_{a}$, it does not become the lowest frequency excitation in the separation range studied (Figure $4 \mathrm{~B}) . \Omega_{\mathrm{L} b}(4 \mathrm{~A})$ in Figure 3 has small interchain coherences. The effect of the phenyl-vinyl contact is illustrated in panel $\Omega_{\mathrm{L} b}(3.5 \mathrm{~A})$ of Figure 3. Here, the electron-hole pair is localized on the central phenyl of molecule I. The off-diagonal intermolecular coherences are different compared to $\Omega_{\mathrm{L} a}$. The phenyl $\rightarrow$ vinyl hole transfer is preferable to the electron-transfer process. This means that $\mathrm{L} b$ is a chargetransfer excitation.

D. PPV-Dimer: case 2 (maximum contact). Since the whole molecule is involved in a contact in this case, delocalized (1-4) and localized (L) monomeric states should interact equally, in contrast to case 1 . We will, therefore, focus on the lowest delocalized transitions 1-4. Figure 5A shows the variation of frequencies $\Omega_{1 a}^{\prime}$ and $\Omega_{1 a}^{\prime \prime}$, corresponding to the band gap transition 1 of case 2 (a). At large separations $d \gtrsim 4 \AA$ their behavior can again be described by a simple exciton model. However, the splitting between the two excited states (Figure 5C) implies a stronger Coulomb interaction compared to case 1. The parallel orientation of the chromophores results in an H-type dimerization (the blue component gains double oscillator 


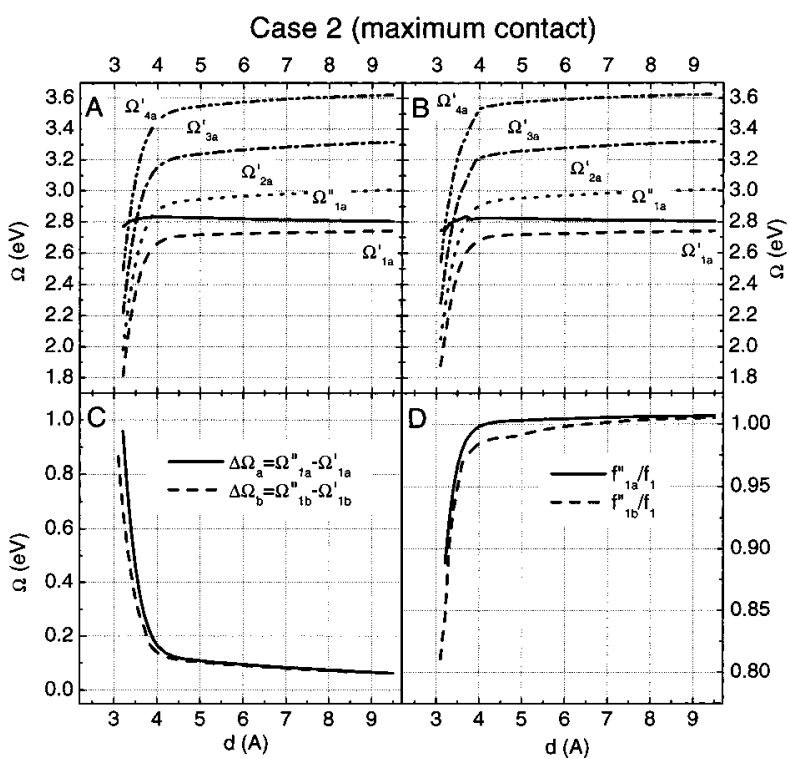

Figure 5. Variation of excited-state energies of $\Omega_{1}^{\prime}-\Omega_{4}^{\prime}$ transitions (panels A and B); energy splitting and relative oscillator strength for band-gap pair of dimer excited states (panels $\mathrm{C}$ and $\mathrm{D}$ ) with separation of PPV chains for (a) and (b) structures of case 2 (Figure 1).

strength, whereas the red component is dark) contrary to J-type aggregation (the oscillator strength goes to the red component and the blue component is optically forbidden). State $1 a^{\prime \prime}$ has the entire oscillator strength, which is conserved (Figure 5D).

At close intermolecular distances $d<4 \AA$, $\Omega_{1 a}^{\prime}$ exhibits a strong red-shift, whereas $\Omega_{1 a}^{\prime \prime}$ does not change significantly (Figure 5A). The splitting therefore rapidly increases up to 0.9 $\mathrm{eV}$ at $d=3.2 \AA$. Electronic mode plots immediately identify the reason for such behavior. Panels $\Omega_{1 a}^{\prime}$ and $\Omega_{1 a}^{\prime \prime}$ in Figure 6 display $1 a^{\prime}$ and $1 a^{\prime \prime}$ modes for $d=3.5 \AA$ intermolecular separation. Red component $1 a^{\prime}$ with vanishing oscillator strength shows strong off-diagonal coherence (exciton delocalization), which results in its frequency red-shift. In complete contrast, the strongly allowed blue component $1 a^{\prime \prime}$ has very small interchromophore coherence and its energy therefore does not change. However, this transition starts losing its oscillator strength at small separations. A similar phenomenon was observed in bridged naphthalene dimers ${ }^{59}$ where the lowest frequency electronic mode also acquires much stronger coherences than its blue counterpart.

Higher frequency transitions (2-4) exhibit the same trends. Panels $\Omega_{2 a}^{\prime}, \Omega_{3 a}^{\prime}$ and $\Omega_{4 a}^{\prime}$ in Figure 6 show contour plots of these modes at $d=3.5 \AA$. Similar to $1 a^{\prime}$, they all have very strong interchromophore coherence. It is interesting to note that offdiagonal parts have the same pattern of coherence distribution as the diagonal area on each plot. This means that any carbon atom $i$ on the molecule I and its symmetric counterpart $i+54$ have similar induced bond orders with other atoms of the aggregate. Finally $\Omega_{L a}$ in Figure 6 displays the formation of an interchain localized transition. Even though the off-diagonal coherences are strong, its energy is still well above the absorbing $1 a^{\prime \prime}$ state. Due to strong intermolecular coherences, $\Omega_{2 a}^{\prime}-\Omega_{4 a}^{\prime}$ show large red-shift and they cross $\Omega_{1 a}^{\prime \prime}$ at close separations. This results in the formation of several forbidden interchain states lying below the absorbing $1 a^{\prime \prime}$ transition.

Case 2(b) (see Figure 5B-D) has analogous trends, although the phenyl-vinyl coherent interaction is weaker than that of the phenyl-phenyl one. We also notice that the lack of symmetry in case 2(b) leads to larger mode mixing (e.g. the curve $\Omega_{1 b}^{\prime \prime}$ in Figure $5 \mathrm{~B}$ is no longer smooth, but rather has small kinks where it is crossed by other states).

\section{E. PPV-dimer: case 3 (intermediate contact)}

Finally, case 3(a) shows intermediate behavior. At the large intermolecular distances $d>4 \AA$ the aggregate is in the Frenkel exciton model (electrostatic) regime (Figure 7A). Here, the splitting $\Delta \Omega_{a}$ is very small (Figure 7B) compared to case 2 and even case 1 , since this intermolecular orientation is close to the crossover to the J-type aggregation. ${ }^{45}$ At smaller separations the dimer exhibits coherent behavior. Panels $\Omega_{1 a}^{\prime}$ and $\Omega_{1 a}^{\prime}$ for case 3 in Figure 6 display $1 a^{\prime}$ and $1 a^{\prime \prime}$ modes for $d=3.2$ $\AA$ intermolecular separation. Similar to the case 2 , the $1 a^{\prime}$ component shows strong off-diagonal coherence and its frequency exhibits red-shift, whereas the $1 a^{\prime \prime}$ mode has very small interchromophore coherence and its energy is stable. We also note that the $1 a^{\prime}$ electron-hole pair tends to be more localized to the contact location so as to favor interchromophore delocalization.

Other higher frequency $\Omega_{a}^{\prime}$ components of transition pairs undergo strong red-shifts at $d<4 \AA$ (Figure 7A), cross the $1 a^{\prime \prime}$ transition and become low-frequency forbidden states. However, since only part of the oligomer is involved in a contact, they shift to the red at a different rate. For example, $3 a^{\prime}$ ultimately has lower energy then $2 a^{\prime}$. In addition, $3 a^{\prime}$ shows significant mixing to the $1 a^{\prime \prime}$ upon their crossing (odd-odd node modes coupling). This is reflected as strong kinks on the $\Omega_{1 a}^{\prime \prime}$ curve (Figure 7A) and redistribution of oscillator strength between $3 a^{\prime}$ and $1 a^{\prime \prime}$ displayed on Figure 7C. On the other hand, electronic modes with an even number of nodes $\left(2 a^{\prime}\right.$ and $\left.4 a^{\prime}\right)$ do not mix with $1 a^{\prime}$ upon crossing. Contour plots of $2 a^{\prime}-4 a^{\prime}$ modes at $d=3.5 \AA$, displayed in panels $\Omega_{2 a}^{\prime}-\Omega_{4 a}^{\prime}$ in Figure 6 (case 3), show very strong interchromophore coherences in the contact region. Compared to the case 2(a), the diagonal patterns of these modes are significantly distorted from their monomer origins, and assignment of these transitions is not straightforward.

In the case 3(b) the dimer first shows J-type behavior, where the absorbing state $1 b^{\prime \prime}$ is the lowest. This crossover from $\mathrm{H}$ to J-type aggregation was explored in ref 45. However, the forbidden $1 a^{\prime}$ transition immediately becomes the lowest as soon as coherent interaction is activated at close separations (see Figure $5 B-D)$. Otherwise this system behaves similarly to the case 3(a).

\section{Discussion}

We have identified two different types of chromophore interactions. At large separations $d \gtrsim 4 \AA$ the chromophores interact through the long-range electrostatic, or dipole-dipole, coupling. Any transition splits into two, and the splitting is the measure of interchromophore coupling. The dimer wave functions are symmetric and antisymmetric combinations of the monomer wave functions. The Frenkel exciton Hamiltonian would be the appropriate model description for these aggregates. ${ }^{61-64}$ Short-range electron-exchange, or a through space interaction which increases exponentially with interchromophore distance, prevails at small intermolecular separations $d \lesssim 4 \AA$. The aggregate wave functions generally can then not be expanded into monomeric states. This interaction leads to the formation of new interchain states and causes an overall redshift of the absorption and the emission.

To characterize intra- and inter-chromophore electron exchange for molecular excitons we can define the probabilities of different processes. Let $p_{11}\left(p_{22}\right)$ be the probability for both electron and hole to reside on monomer I (II). Probabilities for the electron and the hole to be on different chains (charge 

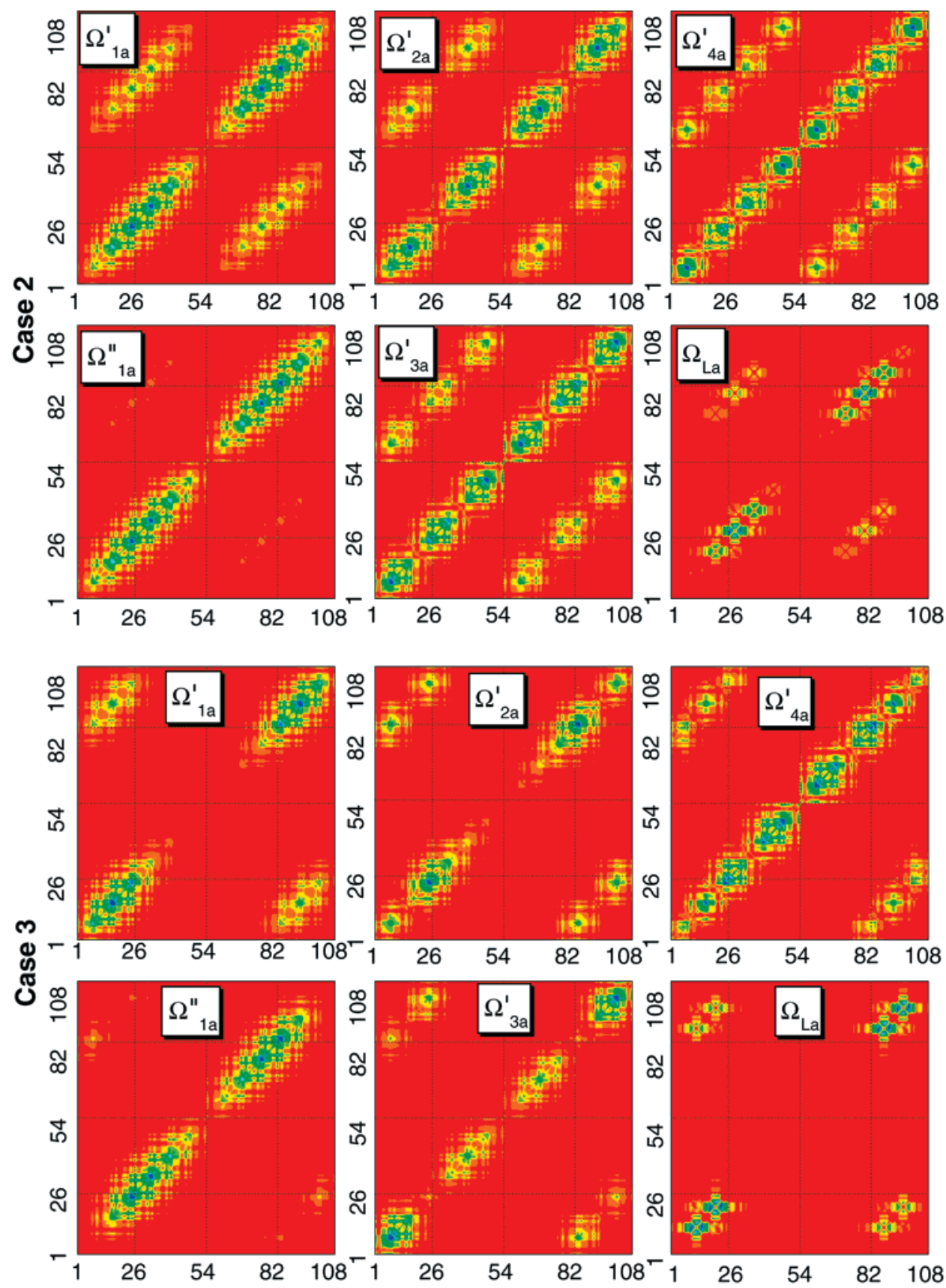

Figure 6. Contour plots of the electronic modes of PPV-7 dimers for case 2 (top two rows) and for case 3 (bottom two rows) at $3.5 \AA$ separation. The axis labels represent the individual atoms, as shown in Figure 1. The color map is given in the middle row of Figure 3 . The dimer modes are labeled according to Figures 1, 5, and 7. Their frequencies are $\Omega_{1 a}^{\prime}=2.36 \mathrm{eV}, \Omega_{1 a}^{\prime \prime}=2.82 \mathrm{eV}, \Omega_{2 a}^{\prime}=2.54 \mathrm{eV}, \Omega_{3 a}^{\prime}=2.80 \mathrm{eV}, \Omega_{4 a}^{\prime}=3.09 \mathrm{eV}$, $\Omega_{\mathrm{L} a}=3.27 \mathrm{eV}$ (case 2) and 2.25, 2.73, 2.92, 3.08, 3.53, $3.00 \mathrm{eV}$ (case 3), respectively.

transfer) are $p_{21}$ and $p_{12}$ (see top panel in Figure 8). These variables can be computed by further contraction of electronic modes to $2 \times 2$ matrices:

$$
p_{i j}=\sum_{n_{i}, m_{j}}\left[\left(\xi_{v}\right)_{n_{i} m_{j}}\right]^{2}, \quad i, j=1,2
$$

with normalization condition $\sum_{i, j} p_{i j}=1$. Here the indices $n_{i}$ and $m_{j}$ run over atomic orbitals localized on monomers $i$ and $j$, respectively. Now $p_{i j}$ are total intra- and intermolecular contributions.

The variation of $p$ with interchain distance $d$ is shown in Figure 8. In case 1(a) the extended excitation (off-diagonal) contributions $p_{12}$ and $p_{21}$ do not exceed 0.1 for all considered separations, whereas the charge transfer (electron exchange) for the localized state $L a$ saturates at 0.4 for $d<3.5 \AA$. $L b$ in case 1(b) has large phenyl $\rightarrow$ vinyl hole-transfer probability, which again saturates to 0.4 . On the other hand, the phenyl $\rightarrow$ vinyl electron-transfer saturates to 0.13 . This assumes that hole transfer is more preferable by a factor of $\sim 3$ for case 1(b) intermolecular orientation. The behavior of the intermolecular probabilities for red-components of low-frequency states in cases 2 and 3 is very similar: the electron exchange quickly increases to 0.4 with decreasing $d$, whereas $p_{12}$ of $1^{\prime \prime}$ state, which has all the oscillator strength, is relatively small $(\sim 0.1)$ for a wide range of $d$ (Figure 8). These results are consistent with studies of excimer formation in PPV derivatives using tight binding band model ${ }^{42}$ which report the probability of the charge-transfer contribution to the excimer wave function increases to 0.4 for distances closer $4 \AA$. 


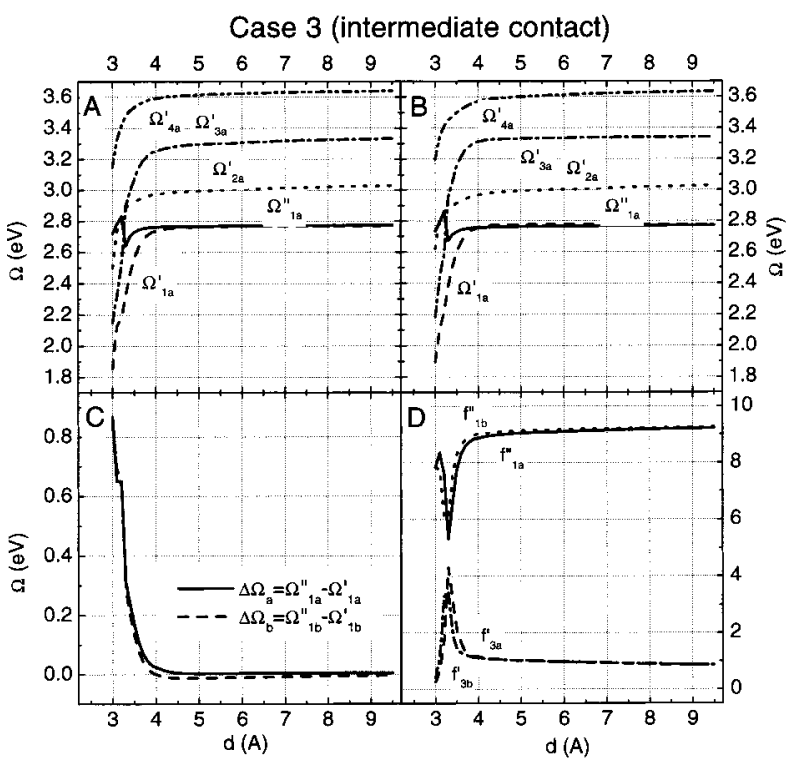

Figure 7. Variation of excited-state energies of $\Omega_{1}^{\prime}-\Omega_{4}^{\prime}$ transitions (panels $\mathrm{A}$ and $\mathrm{B}$ ); energy splitting for band-gap pair of dimer excited states (panel C); oscillator strengths of 1 and 3 excited states (panel D) with separation of PPV chains for (a) and (b) structures of case 3 (Figure 1).

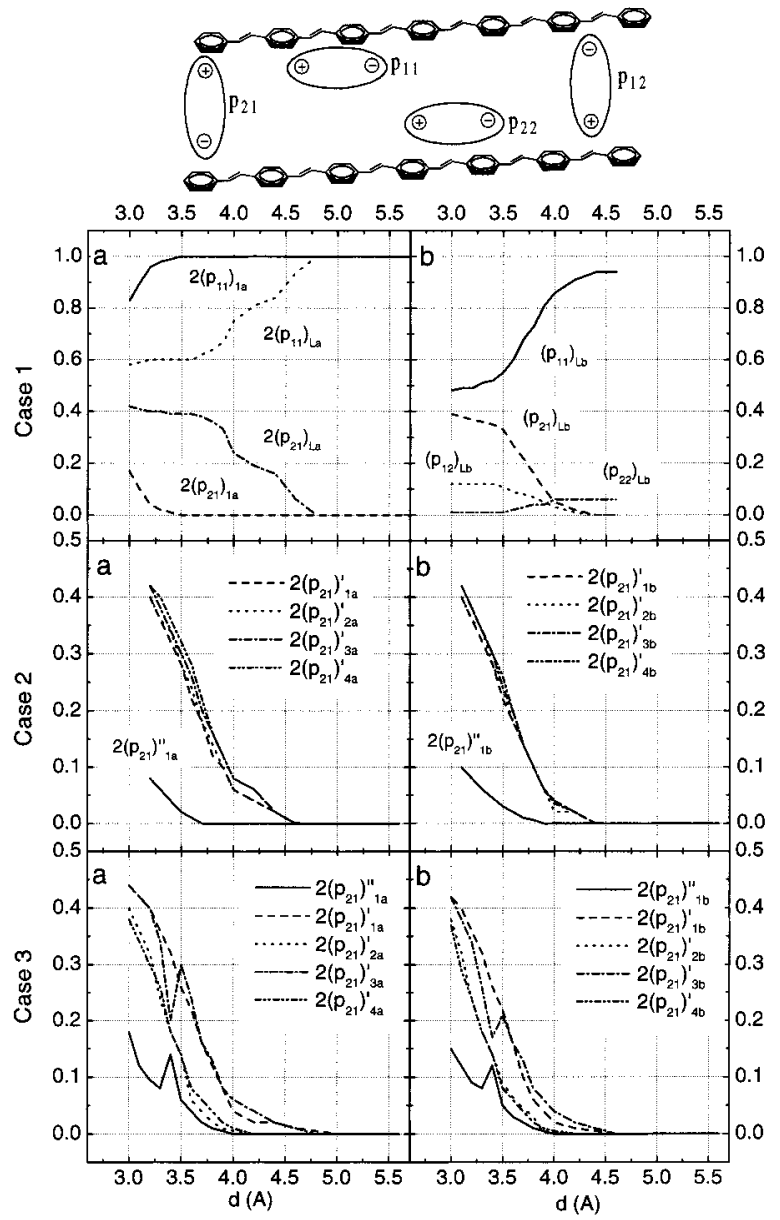

Figure 8. Top panel: representation of four exciton positions in a PPV dimer. Lower panels: Variation of probabilities for processes shown in the top panel for low-frequency excited states in aggregates (case 1-3) with separation of PPV chains.

Although the mechanisms of interchain excitation formation in cases $1-3$ are different, the resulting physical picture is the same, as shown in Figure 8. All these excitations have vanishing oscillator strength, very strong intermolecular electron exchange (coherence), and their frequencies are extremely sensitive to the interchain separation. On the other hand, the principal absorbing states show very small intermolecular electron exchange and their frequencies do not vary significantly with intermolecular separation. The mutual orientation of the PPV chains in polymer films may lead to a distribution of intermolecular orientations. Low-lying intermolecular states form for any contacts closer than $\sim 4 \AA$ which may account for the reduced photoluminescence in these materials.

Acknowledgment. The authors thank V. I. Klimov and D. W. McBranch for helpful discussions. This work was performed under the auspices of the U.S. Department of Energy and the LDRD program at LANL. S.T. gratefully acknowledges the support of a LANL Director's Postdoctoral Fellowship. The numerical computations were performed using the resources of the Center for Nonlinear Studies at Los Alamos National Laboratory.

\section{References and Notes}

(1) Burroughes, J.; Bradley, D. D. C.; Brown, A.; Marks, R.; Mackay, K.; Friend, R.; Burns, P.; Holmes, A. Nature 1990, 347, 539.

(2) Braun, D.; Heeger, A. J. Appl. Phys. Lett. 1991, 58, 1982.

(3) Pei, Q. B.; Yu, G.; Zhang, C.; Yang, Y.; Heeger, A. J. Science 1995, 269, 1086

(4) Yu, G.; Gao, J.; Hummelen, J. C.; Wudl, F.; Heeger, A. J. Science 1995, 270,1789

(5) Sirringhaus, H.; Tessler, N.; Friend, R. H. Science 1998, 280, 1741.

(6) Ho, P. K. H.; Thomas, D. S.; Friend, R. H.; Tessler, N. Science 1999, 285,233 .

(7) Dodabalapur, A.; Bao, Z.; Makhja, A.; Laquindanum, J. C.; Raju, V. R.; Feng, Y.; Katz, H. E.; Rogers, J. Appl. Phys. Lett. 1998, 73, 142

(8) Yu, G.; Pakbaz, K.; Heeger, A. J. Appl. Phys. Lett. 1994, 64, 3422.

(9) Yu, H.; Hsieh, B. R.; Abkowitz, M. A.; Jenekhe, S. A.; Stolka, M. Synth. Met. 1994, 62, 265.

(10) Katz, H. E. J. Mater. Chem. 1997, 7, 369

(11) Gundlach, D. J.; Lin, Y. Y.; Jackson, T. N.; Schlom, D. G. Appl. Phys. Lett. 1997, 71, 3853

(12) Hide, F.; Diaz Garsia, M. A.; Schwartz, B. J.; Andersson, M. R.; Pei, Q. B.; Heeger, A. J. Science 1996, 273, 1833.

(13) Tessler, N.; Denton, G. J.; Friend, R. H. Nature 1996, 382, 695

(14) Frolov, S. V.; Shkunov, M.; Vardeny, Z. V.; Yoshino, K. Phys. Rev. B 1997, 56, R4363.

(15) Jenekhe, S. A.; Osaheni, J. A. Science 1994, 265, 765

(16) Conwell, E. Trends Polym. Sci. 1997, 5, 218.

(17) Jakubiak, R.; Collison, C. J.; Chou Wan, W.; Hsieh, B. R.; Rothberg,

L. J. J. Phys. Chem. A 1999, 103, 2394.

(18) Yan, M.; Rothberg, L. J.; Papadimitrakopoulos, F.; Galvin, M. E.; Miller, T. Phys. Rev. Lett. 1994, 73, 744.

(19) Yan, M.; Rothberg, L. J.; Kwock, E. W.; Miller, T. M. Phys. Rev. Lett. 1995, 75, 1992.

(20) McBranch, D. W.; Sinclair, M. B. In The Nature of the Photoexcitations in Conjugated Polymers; Sariciftci, N. S., Ed.; Word Scientific Publishing: Singapore, 1997.

(21) Sun, B. J.; Miao, Y.-J.; Bazan, G. C.; Conwell, E. M. Chem. Phys Lett. 1996, 260, 186.

(22) Gettinger, C. L.; Heeger, A. J.; Drake, J. M.; Pine, D. J. J. Chem. Phys. 1994, 101, 1673.

(23) Hsieh, B. R.; Yu, Y.; Forsythe, E. W.; Schaaf, G. M.; Feld, W. A. J. Am. Chem. Soc. 1998, 120, 231.

(24) Yan, M.; Rothberg, L. J.; Papadimitrakopoulos, F.; Galvin, M. E.; Miller, T. M. Phys. Rev. Lett. 1994, 72, 1104.

(25) Klimov, V. I.; McBranch, D. W.; Barashkov, N. N.; Ferraris, J. P. Chem. Phys. Lett. 1997, 277, 109.

(26) Samuel, I. D. W.; Rumbles, G.; Collison, C. J.; Moratti, S. C.; Holmes, A. B. Chem. Phys. 1998, 227, 75.

(27) Klimov, V. I.; McBranch, D. W.; Barashkov, N. N.; Ferraris, J. P. Phys. Rev. B 1998, 58, 7654

(28) Leng, J. M.; Jeglinskii, S.; Wei, X.; Benner, R. E.; Vardeny, Z V.; Guo, F.; Mazumdar, S. Phys. Rev. Lett. 1994, 72, 156.

(29) Kraabel, B.; Klimov, V. I.; Kohlman, R.; Xu, S.; Wang, H-L.; McBranch, D. W. Phys. Rev. B 2000, 61, 8501.

(30) Rauscher, U.; Bässler, H.; Göbel, E. O. Phys. Rev. B 1990, 42, 9830 
(31) Greenham, N. C.; Samuel, I. D. W.; Hayes, G. R.; Kessener, Y.; Moratti, S. C.; Holmes, A. B.; Friend, R. H. Chem. Phys. Lett. 1995, 241, 89.

(32) Kirova, N.; Brazovskii, S.; Bishop, A. R. Opt. Mater. 1999, 100, 29

(33) Gartstein, Yu. N.; Rice, M. J.; Conwell, E. M. Phys. Rev. B. 1995, $51,5546$.

(34) Gartstein, Yu. N.; Rice, M. J.; Conwell, E. M. Phys. Rev. B. 1995, $52,1683$.

(35) Brédas, J. L.; Cornil, J.; Beljonne, D.; Dos Santos, D. A.; Shuai, Z. Acc. Chem. Res. 1999, 32, 267.

(36) Marder, S. R.; Kippelen, B.; Jen, A. K.-Y.; Peyghambarian, N. Nature 1997, 388, 845.

(37) Mukamel, S.; Tretiak, S.; Wagersreiter, T.; Chernyak, V. Science 1997, 277,781 .

(38) Brédas, J. L.; Adant, C.; Tackyx, P.; Persoons, A.; Pierce, B. M. Chem. Rev. 1994, 94, 243.

(39) Kanis, D. R.; Ratner, M. A.; Marks, T. J. Chem. Rev. 1994, 94, 195.

(40) Yu, Z. G.; Wu, M. W.; Rao, X. S.; Bishop, A. R. J. Phys.: Condens. Matter 1996, 8, 8847.

(41) Yu, Z. G.; Saxena, A.; Bishop, A. R. Phys. Rev. B. 1997, 56, 3697.

(42) Wu, M. W.; Conwell, E. M. Phys. Rev. B 1997, 56, R10060.

(43) Conwell, E. M. Phys. Rev. B 1998, 57, 14200.

(44) Cornil, J.; Heeger, A. J.; Brédas, J. L. Chem. Phys. Lett. 1997, $272,463$.

(45) Siddiqui, S.; Spano, F. C. Chem. Phys. Lett. 1999, 308, 99.

(46) Conwell, E. M.; Perlstein, J.; Shaik, S. Phys. Rev. B 1996, 54, R2308.

(47) Bazan, G. C.; Oldham, W. J., Jr.; Lachicotte, R. J.; Tretiak, S.; Chernyak, V.; Mukamel, S. J. Am. Chem. Soc. 1998, 120, 9188.

(48) Tretiak, S.; Chernyak, V.; Mukamel, S. J. Am. Chem. Soc. 1997, 119,11408

(49) Tretiak, S.; Chernyak, V.; Mukamel, S. J. Phys. Chem. B 1998 , 102,3310
(50) Dewar, M. J. S.; Zoebisch, E. G.; Healy, E. F.; Stewart, J. J. P. J Am. Chem. Soc. 1985, 107, 3902.

(51) Tretiak, S.; Chernyak, V.; Mukamel, S. J. Chem. Phys. 1996, 105, 8914.

(52) Chernyak, V.; Tretiak, S.; Schulz, M.; Tsiper, E. V.; Mukamel, S. J. Chem. Phys. 2000, 113, 36.

(53) Pople, J. A.; Segal, G. A. J. Chem. Phys. 1965, 43, S136.

(54) Pople, J. A.; Beveridge, D. L.; Dobosh, P. J. Chem. Phys. 1967, 47, 2026.

(55) Ridley, J.; Zerner, M. C. Theor. Chim. Acta 1973, 32, 111.

(56) Zerner, M. C.; Loew, G. H.; Kirchner, R. F.; Mueller-Westerhoff,

U. T. J. Am. Chem. Soc. 1980, 102, 589.

(57) McWeeny, R.; Sutcliffe, B. T. Methods of Molecular Quantum Mechanics; Academic Press: New York, 1976.

(58) Davidson, E. R. Reduced Density Matrices in Quantum Chemistry; Academic Press: New York, 1976.

(59) Tretiak, S.; Zhang, W. M.; Chernyak, V.; Mukamel, S. Proc. Nat. Acad. Sci. U.S.A. 1999, 96, 13003.

(60) Fukutome, H. J. Mol. Struct. (THEOCHEM) 1989, 188, 337.

(61) Pope, M.; Swenberg, C. E. Electronic Processes in Organic Crystals; Clarendon Press (Oxford University Press): Oxford, New York, 1982.

(62) Silinsh, E. A.; Čápek, V. Organic Molecular Crystals. AIP Press: American Institute of Physics, New York, 1994.

(63) Rashba, E. I.; Sturge, M. D., Eds.; Excitons; North-Holland: Amsterdam, 1982.

(64) Broude, V. B.; Rashba, E. I.; Sheka, E. F. Spectroscopy of Molecular Excitons; Springer: Berlin, 1985.

(65) Davydov, A. S. Theory of Molecular Excitons; Plenum: New York, 1971.

(66) Kasha, M.; Rawls, H. R.; Ashraf El-Bayoumi, M. Pure Appl. Chem.

1965, 11, 371.

(67) Poliakov, E.; Chernyak, V.; Tretiak, S.; Mukamel, S. J. Chem. Phys. 1999, 110, 8161 . 